\title{
ARTIGOORIGINAL
}

\section{INFLUÊNCIA DA DEPRESSÃO NA COMPETÊNCIA LEITORA EM ESTUDANTES DE PSICOLOGIA}

DOI: 10.22289/2446-922X.V6N2A11

\author{
Patrícia Maria da Silva Costa ${ }^{1}$ \\ Thiago da Silva Gusmão Cardoso \\ Damares Borges dos Santos \\ Elisabete Agrela de Andrade
}

\section{RESUMO}

É crescente a prevalência de transtornos mentais na população universitária, principalmente, depressão, ansiedade e estresse. Partindo do princípio de que a vulnerabilidade em saúde mental pode refletir na aprendizagem dos alunos, o presente estudo buscou compreender como os sintomas de depressão interferem na capacidade de compreensão leitora de estudantes universitários. Trata-se de estudo transversal quantitativo, composto por uma amostra de 96 discentes do $1^{\circ}$ e $5^{\circ}$ ano do Curso de Psicologia. Foram utilizados: Inventário de Depressão de Beck, Teste de Cloze e questionário sociodemográfico. Os dados foram tratados por meio de análise estatística descritiva e inferencial. Houve correlação inversa entre a intensidade dos sintomas de depressão com as habilidades em leitura nos concluintes $(r=-0,3)$, entre os ingressantes uma correlação direta não significante $(r=0,1)$. Conclui-se que existe influência dos sintomas de depressão sobre as dificuldades na compreensão leitora, o que mostra a necessidade de atenção à saúde mental de estudantes, especialmente no período final do curso de Psicologia. Entre estudantes de Psicologia, esta situação pode trazer influências para sua futura atuação profissional.

Palavras-chave: Depressão; Saúde do Estudante; Saúde Mental.

\section{INFLUENCE OF DEPRESSION ON READING COMPETENCE IN PSYCHOLOGY STUDENTS}

\section{ABSTRACT}

The prevalence of mental disorders in the university population is increasing, mainly depression, anxiety and stress. Assuming that vulnerability in mental health can reflect on students' learning, the present study sought to understand how depression symptoms interfere with the reading comprehension ability of university students. This is a quantitative cross-sectional study, composed of a sample of 96 students from the 1st and 5th year of the Psychology Course. Beck Depression Inventory, Cloze Test and sociodemographic questionnaire were used.

\footnotetext{
${ }^{1}$ Endereço eletrônico de contato: patricia.maria@unasp.edu.br

Recebido em 21/05/2020. Aprovado pelo conselho editorial para publicação em 17/09/2020.
} 
The data were treated using descriptive and inferential statistical analysis. There was an inverse correlation between the intensity of the symptoms of depression and reading skills in the graduates $(r=-0.3)$, among the freshmen a direct non-significant correlation $(r=0.1)$.

It is concluded that there is an influence of the symptoms of depression on the difficulties in reading comprehension, which shows the need for attention to the mental health of students, especially in the final period of the Psychology course. Among psychology students, this situation can influence their future professional performance.

Keywords: Depression; Student Health; Mental Health.

\section{INFLUENCIA DE LA DEPRESIÓN EN LA COMPETENCIA LECTORA EN ESTUDIANTES DE PSICOLOGÍA}

\section{RESUMEN}

La prevalencia de los trastornos mentales en la población universitaria está aumentando, principalmente depresión, ansiedad y estrés. Basado en el principio de que la vulnerabilidad en la salud mental puede reflejarse en el aprendizaje de los estudiantes, el presente estudio buscó comprender cómo los síntomas de depresión interfieren en la capacidad de comprensión lectora de los estudiantes universitarios. Este es un estudio cuantitativo de corte transversal, compuesto por una muestra de 96 estudiantes del primer y quinto año del curso de psicología. Se utilizaron el Inventario de Depresión de Beck, la Prueba Cloze y el cuestionario sociodemográfico. Los datos fueron tratados mediante análisis estadístico descriptivo e inferencial. Hubo una correlación inversa entre la intensidad de los síntomas de depresión y las habilidades de lectura en los graduados $(r=$ -0.3), entre los estudiantes de primer año una correlación directa no significativa $(r=0.1)$. Se concluye que existe una influencia de los síntomas de la depresión en las dificultades en la comprensión lectora, lo que demuestra la necesidad de prestar atención a la salud mental de los estudiantes, especialmente en el período final del curso de Psicología. Entre los estudiantes de Psicología, esta situación puede influir en su futuro desempeño professional.

Palabras clave: Depresión; Salud del Estudiante; Salud Mental.

\section{INTRODUÇÃO}

Segundo a Organização Mundial da Saúde aproximadamente 300 milhões de pessoas no mundo sofrem de depressão (WHO, 2019). O aumento da prevalência de transtornos depressivos no mundo vem crescendo e está relacionado às desigualdades sociais, adoecimento da população e situações da cultura contemporânea. Sua alta taxa de prevalência faz com que seja considerada um transtorno comum, entretanto sua sintomatologia envolve sintomas psicológicos e fisiológicos prejudiciais à qualidade de vida do indivíduo.

De acordo com o DMS-5, para diagnosticar a depressão, é necessário que no período que compreende duas semanas ou mais, o indivíduo apresente humor deprimido, perda de interesse e/ou prazer, alterações no peso (aumento ou diminuição), alterações no sono, cansaço, perda de energia, dificuldades com a concentração, alterações psicomotoras, sentimentos de desvalia, culpa, 
pensamentos recorrentes de morte e ideação suicida (APA, 2014). Não há uma especificidade de fase ou idade para o surgimento da depressão, podendo estar relacionado a fatores genéticos, fisiológicos, ambientais, temperamento e comorbidades, os quais irão se manifestar no físico, emocional, influenciando os processos cognitivos, nas funções vegetativas, e também nos relacionamentos interpessoais do indivíduo (Costa, 2015).

Considerando o ambiente escolar, o desempenho dos alunos, no contexto da aprendizagem, está associado a diversos fatores condicionantes, sendo a afetividade, a principal característica determinante ao desenvolvimento do sujeito. Sob a perspectiva de que o ensino básico, fundamental e médio forma a base para a continuidade do aprendizado na vida acadêmica (BNCC, 2019), é assustador a frequência em que a literatura aponta a prevalência de depressão, estresse e ansiedade em crianças no período escolar, indicando a influência que as emoções e a saúde, provocam no desenvolvimento escolar (Garcia \& Boruchovitch, 2015).

Garcia e Boruchovitch (2015) realizaram uma pesquisa com 275 alunos do $5^{\circ}$ ao $9^{\circ}$ ano de uma escola pública, em Minas Gerais - MG, no ano de 2012, para identificar as atribuições de causalidade para o sucesso e fracasso escolar, bem como verificar se existe relação entre essas atribuições e variáveis demográficas e escolares. Os dados foram coletados por meio de questionário demográfico e Escala de Avaliação das Atribuições de Causalidade para sucesso e fracasso escolar, indicando, num contexto geral, que os alunos atribuíram a causas internas e controláveis, os motivos de seus sucessos e fracassos escolares.

O estudo citado demonstra uma relação entre sintomas emocionais e prejuízo acadêmico, principalmente, no desenvolvimento das habilidades em leitura, escrita e matemática, sendo atribuído pelos próprios alunos o fracasso escolar a fatores internos (esforço e capacidade). Desta forma, os sintomas de depressão, ao impactarem sobre o autoconceito e às atribuições de causalidade para o sucesso e fracasso escolar, podem ser fatores de risco ao desenvolvimento de dificuldades de aprendizagem em crianças da educação básica. Entretanto, há uma escassez de estudos em outras etapas do ensino, como no superior.

Papalia e Feldman (2013) apontam que a etapa do ensino superior é marcada por intensas mudanças e adaptações, tanto na vida pessoal como acadêmica do aluno. Diversos fatores contribuem para o sucesso acadêmico no ensino superior, por exemplo, as práticas de leitura influenciam o êxito ou fracasso acadêmico, pois afetam diretamente o nível de aprendizagem dos alunos (Tanzawa \& Pullin, 2012). Desse modo, fez-se necessário perceber a maneira que a depressão interfere na aprendizagem e na compreensão/habilidades em leitura.

Mediante os diversos fatores que influenciam a saúde mental do aluno universitário, Borine, Wanderley e Bassit (2015) apresentam que as experiências universitárias tendem a gerar sofrimento psíquico ao aluno, graças ao constante estresse vivenciado, associado às demandas universitárias. Sendo a leitura constituída por processos cognitivos (Stenberg, 2012) que dependem 
da dimensão afetiva, a discussão desta pesquisa é que desequilíbrios afetivos e emocionais, como o transtorno depressivo, que ocasionam prejuízos a saúde mental do indivíduo, podem interferir na aprendizagem dos alunos universitários, podendo ser detectada por meio de dificuldades na compreensão em leitura.

Sob esse olhar, o presente artigo tem como objetivo compreender como o fenômeno da depressão se reproduz nesta outra etapa educacional, tendo em vista o que a literatura aponta sobre isso no período escolar, bem como à necessidade do aprofundamento de estudos relacionados a saúde mental dos universitários, considerando a vulnerabilidade em que se encontram estes jovens.

\section{MATERIAIS E MÉTODOS}

Trata-se de um estudo transversal quantitativo, que investigou através de inventários e testes de desempenho, a relação entre sintomas de depressão e a competência leitora.

Participantes

A amostra foi constituída pelos alunos do curso de Psicologia de um centro universitário

privado de São Paulo - SP. A seleção da população seguiu princípios não probabilísticos, sendo intencional. Foram escolhidos os alunos ingressantes ( $1^{\underline{a}}$ etapa) e concluintes ( $9^{\underline{a}}$ etapa e $10^{\underline{a}}$ etapa) a fim de analisar se a entrada bem como permanência no ensino superior pode influenciar a saúde mental do indivíduo (sintomas de depressão), relacionando com os níveis de compreensão em leitura entre estes dois grupos.

\section{Procedimentos}

A fim de analisar as variáveis relacionadas a saúde mental e habilidades em leitura, foi desenvolvido para o referido estudo um questionário de caracterização sociodemográfico que abrangia questões relacionadas a saúde mental e leitura. O instrumento possui 22 questões, abarcando os seguintes dados: período de curso; gênero; perfil etário; estado civil; religião; categoria administrativa da instituição de ensino de conclusão do ensino fundamental e médio; exercício de atividade remunerada e tempo diária; diagnóstico de depressão; uso da psicoterapia pessoal e tempo; utilização de medicamentos e tipo; diagnóstico de problemas de aprendizagem; dificuldades com a leitura e escrita; hábitos de leitura.

Rev. Psicol Saúde e Debate. Out., 2020:6(2): 148-161. 
Para verificar a intensidade e presença dos sintomas do transtorno depressivo, por meio de uma avaliação quantitativa, foi aplicado o Inventário de Depressão de Beck (BDI II), um questionário padronizado autoaplicável, que se configura como uma medida de avaliação dos sintomas de depressão. A classificação dos sintomas é dividida entre 0-11 (mínimo), 12-19 (leve), 20-35 (moderado), 36-63 (grave), segundo Goresteie e Wang (2016). Na literatura é encontrado diversos estudos que atestam a confiabilidade do instrumento para mensurar a sintomatologia da depressão entre os universitários (Bolsoni-Silva \& Guerra, 2014; Estrada Aranda, Álvarez, Hernández \& Ramírez, 2015).

No presente estudo, foi utilizado também o teste de Cloze - Labirinto: a estruturação de um texto de aproximadamente 250 palavras, no qual são omitidos os quintos vocábulos, cabendo ao indivíduo preencher as lacunas referentes às omissões derivadas da escolha de palavras adequadas ao contexto apresentado, afim de mensurar as habilidades de compreensão em leitura por meio da análise da compreensão crítica e fonológica do texto (Abreu, Garcia, Hora \& Souza, 2017).

A coleta de dados foi realizada no mês de maio de 2018, cuja população inicial era constituída por 99 alunos do curso de Psicologia, sendo 49 ingressantes e 50 concluintes de um centro universitário privado de São Paulo - SP, mas apenas 96 alunos cumpriram com todos os protocolos para permanência da pesquisa, foram excluídos três participantes concluintes por não cumprirem com todos os protocolos para permanência na pesquisa.

\section{Análise de Dados}

Todos os dados coletados foram tabulados e analisados com o auxílio do Software Microsoft Office Excel 2010. Os dados foram analisados por meio de testes de correlação de Pearson, através do programa GraphPad Prism6.

\section{Aspectos Éticos da Pesquisa}

A pesquisa foi aprovada pelo Comitê de Ética em Pesquisa, Parecer Plataforma Brasil 2.246.176. A participação da pesquisa foi voluntária e autorizada pelo estudante mediante a assinatura do Termo de Consentimento Livre e Esclarecido, que lhe garantiu o sigilo, a desistência da pesquisa sem que isto lhe causasse algum prejuízo, o acolhimento e a orientação de atendimento psicológico nos serviços de saúde se fosse identificada a necessidade por parte do participante. 


\section{RESULTADOS}

Os resultados deste estudo serão apresentados em três sessões: perfil da amostra; compreensão em leitura em universitários e a presença dos sintomas de depressão entre a população universitária.

Sobre o Perfil sociodemográfico da amostra: dos 96 participantes, 85,4\% eram do sexo feminino e $14,5 \%$ do sexo masculino. Quanto a faixa etária, $69,7 \%$ tinha idade entre 18 e 24 anos e $30,2 \%$ igual ou superior a 25 anos. Referente ao estado civil, $79,1 \%$ eram solteiros, $16,6 \%$ casados e 4,16\% divorciados. Referente a religião, 67,7\% são protestantes, 19,7\% católicos, $1 \%$ evangélicos e 11,4\% informou não possuir religião. Em relação ao exercício de uma atividade remunerada entre os 96 discentes, 48,9\% declararam estar trabalhando atualmente, entre estes, $85,1 \%$ acima de 5 horas diárias e 14,8\% menos de 4 horas diárias.

Com relação a categoria administrativa da instituição de ensino de conclusão do ensino fundamental e médio dos participantes, entre os ingressantes, 38,7\% concluíram o ensino fundamental e 59,1\% o ensino médio em colégio público. Quanto aos concluintes, $59,5 \%$ realizaram o ensino fundamental na rede pública, e 68,0\% o ensino médio.

Referente a saúde mental dos participantes, dos 96 alunos, 16,6\% informou possuir diagnóstico de depressão, sendo 11 ingressantes e 5 concluintes. Sobre o uso de antidepressivos, $8,1 \%$ dos ingressantes faz uso e $8,5 \%$ dos concluintes, sendo citados os seguintes medicamentos: sertralina, desvenlafaxine, escitalipatram e pondera.

Quanto ao acompanhamento psicológico, dos 96 alunos, 31,2\% informaram fazer psicoterapia pessoal, sendo $24,4 \%$ ingressantes e $38,2 \%$ concluintes, destes, $76,6 \%$ o fazem há aproximadamente 12 meses, e $23,3 \%$ há mais de 13 meses. Esta pergunta não objetivou associar a psicoterapia ao adoecimento mental, mas ressaltar sua importância para a formação dos alunos do Curso de Psicologia.

No que concerne as habilidades em leitura, 95,9\% dos ingressantes informaram ter apreço pela leitura, mas $87,7 \%$ declarou considerar insuficiente o tempo que emprega a leitura, destes, $55,1 \%$ informaram possuir dificuldades de leitura, e 55\% informou ter dificuldades para escrever. Quanto aos concluintes, 93,2\% informou ter apreço pela leitura, entre estes $82,9 \%$ considera insuficiente o tempo dedicado a leitura, e 17\% informaram possuir dificuldades no processo de leitura, referente a dificuldades na escrita, apenas 10,6\% informou possuir.

Sobre a compreensão de leitura, na Tabela 1 é apresentada a frequência ( $n$ ) e porcentagem de acertos obtidos no teste de Compreensão de leitura (Cloze) entre ingressantes e concluintes.

Rev. Psicol Saúde e Debate. Out., 2020:6(2): 148-161. 


\section{Tabela 1}

Nível de compreensão demonstrado no teste de Cloze entre ingressantes e concluintes

\section{Frequência}

(N)

\section{Porcentagem entre}

ingressantes
Porcentagem

entre

concluintes

\begin{tabular}{cccc}
\hline $\begin{array}{c}\text { Não houve } \\
\text { compreensão }\end{array}$ & 5 & $8,1 \%$ & $4,2 \%$ \\
Compreensão & 65 & $67,3 \%$ & $65,9 \%$ \\
suficiente & & & \\
Compreensão & 26 & $24,4 \%$ & $29,7 \%$ \\
crítica & & & \\
\hline
\end{tabular}

Produzido pelos autores

A respeito dos resultados obtidos no teste de Cloze, a pontuação máxima possível eram 33 pontos. Os ingressantes alcançaram o máximo de 30 pontos e mínimo de 11 ( $m=22,32 ; d p=4,31)$. O resultado foi similar entre os concluintes, a pontuação máxima foi 31 pontos, e mínimo $11(\mathrm{~m}=$ 23,17; $\mathrm{dp}=4,08$ ). Da amostra total, apenas $1 \%$ informou conhecer o texto Da Timidez, utilizado no Cloze.

Sobre os aspectos relacionados ao aprender, entre os ingressantes 6,1\% da amostra possui diagnóstico de problemas de aprendizagem, não sendo especificado pelos participantes a classificação. Entre estes, 2\% informou possuir diagnóstico de Transtorno do Déficit de Atenção/Hiperatividade (TDAH), não houve nenhum relato sobre dislexia. Enquanto que entre os concluintes, nenhum dos participantes informou possuir diagnóstico de problemas de aprendizagem ou TDAH.

No que diz respeito à presença dos sintomas de depressão, a Tabela 2 apresenta a intensidade dos sintomas de depressão detectados na amostra por meio do Inventário de Depressão de Beck (BDI II). A pontuação obtida foi analisada por meio de quatro categorias, sendo de 0 a 13 pontos (mínimo), de 14 a 19 pontos (leve), de 20 a 28 pontos (moderado) e de 29 a 63 pontos (grave), conforme utilizado em outros estudos (Gorestein \& Wang, 2016; Finger \& Argimon, 2013).

Rev. Psicol Saúde e Debate. Out., 2020:6(2): 148-161. 


\section{Tabela 2}

Intensidade dos sintomas de depressão entre ingressantes e concluintes segundo BDI - II

$\begin{array}{ccc}\text { Frequência } & \text { Porcentagem entre } & \\ \text { (n) } & \text { ingressantes } & \text { Porcentagem entre concluintes }\end{array}$

\begin{tabular}{cccc}
\hline Mínimo & 51 & 38,7 & 68,0 \\
Leve & 25 & 25,5 & 25,5 \\
Moderado & 19 & 32,6 & 6,5 \\
Grave & 1 & 2,0 & 0 \\
\hline
\end{tabular}

Produzido pelos autores

Quanto à análise da presença dos sintomas de depressão e sua relação com a compreensão em leitura, quando realizada a correlação entre a intensidade dos sintomas de depressão por meio do Inventário de Depressão de Beck (BDI II) e o nível de compreensão em leitura obtidos pelo teste de Cloze entre os concluintes, foi encontrada uma correlação inversa e estatisticamente significante, $(r=-0,3)$, quanto maior foi a pontuação alcançada no BDI II menor foram os pontos obtidos no teste de Cloze (ver figura 1).

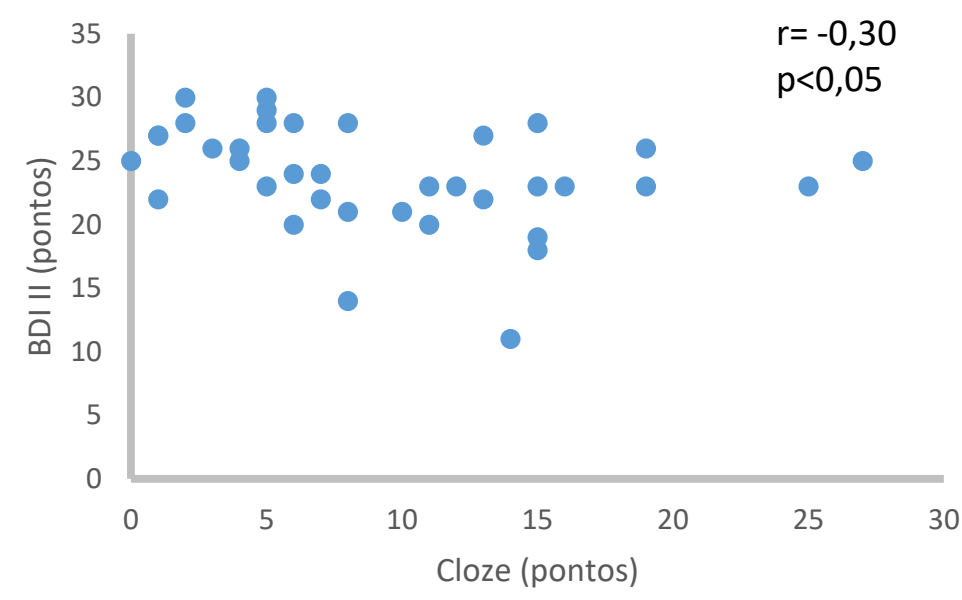

Figura 1 Correlação entre o BDI II e o Cloze entre os alunos concluintes

Entretanto, quando realizada a mesma correlação entre a pontuação obtida no BDI II e pontuação no teste de Cloze entre os ingressantes, foi encontrada uma correlação direta não significante, $(r=0,1, p>0,05)$. 


\section{DISCUSSÃO}

Como demonstrado nos resultados, é significativa a presença dos sintomas de depressão em alunos ingressantes e concluintes.

A intensidade dos sintomas no BDI II evidencia que o aluno ingressa no ensino superior estando já com a saúde mental fragilizada (Bolsoni \& Guerra, 2014). Tal condição, em alguns casos, tende a ser mantida no decorrer da formação acadêmica do aluno, como demonstrado pela intensidade dos sintomas de depressão também apresentados pelos alunos concluintes da referida amostra. De acordo com Paula et al. (2014), a depressão é um dos transtornos com maior prevalência entre os discentes do ensino superior.

Dessa forma, com os alunos já ingressando adoecidos na universidade, é possível pensar na ausência de recursos internos para lidar com as questões que permeiam o momento de vida experienciada. O comprometimento da saúde mental do aluno ingressante está relacionado a diversos fatores, dentre eles a transição da adolescência para a vida adulta, a saída da casa dos pais, maior responsabilidade quanto à vida financeira e administração do tempo, a criação de novos vínculos afetivos interpessoais, entre outros.

Além dos prejuízos ocasionados pelos sintomas da depressão, Moreira, Martins-Reis e Santos (2016) trazem que pesquisas associam o transtorno também ao baixo rendimento acadêmico, tornando válido sua associação a dificuldades no processo de compreensão em leitura, visto que este é um dos requerentes para uma aprendizagem significativa.

Uma questão a ser considerada também, quanto à presença dos sintomas de depressão entre a população universitária, está relacionada a predominância das mulheres entre a amostra $(85,4 \%)$, frente a maior predisposição que as mulheres apresentam para o desenvolvimento do transtorno depressivo se comparado aos homens.

Quanto ao diagnóstico de depressão, os alunos que apresentaram maior pontuação no BDI II informaram possuir diagnóstico de depressão. Entretanto, é significante o nível dos sintomas apresentados por aqueles que informaram não possuir diagnóstico. Este dado pode estar relacionado à ausência de conhecimento por parte do indivíduo no que concerne à saúde mental, ou à desvalorização dos sintomas, resultante dos preconceitos derivados de construções acerca do adoecimento mental.

Sobre o uso de antidepressivos, os dados do presente estudo corroboram com os apresentados pelo Fonaprace (2019), 8,9\% dos alunos das instituições federais informaram já terem feito uso de medicamentos psiquiátricos.

Os resultados obtidos por meio do teste de Cloze demonstraram que os alunos do ensino superior possuem nível de compreensão em leitura abaixo do esperado para o referido nível de escolaridade, conforme tem sido discutido na literatura, visto que $24,4 \%$ dos ingressantes 
compreenderam de maneira crítica o texto, e entre os concluintes foram $29,7 \%$, não havendo diferenças significativas entre os dois grupos.

Predominantemente entre os dois grupos foi informado "sim", quando perguntados se gostavam de ler, porém de maneira proporcional, informaram ser insuficiente o tempo dedicado a leitura, mediante estes dados e aos demais discutidos no presente estudo, a compreensão em leitura envolve aspectos não somente textuais e cognitivos, mas também socioculturais e motivacionais, refletidos nos hábitos de leitura desenvolvidos pelos alunos ingressantes e concluintes no ensino superior.

A partir da perspectiva de Almeida, Piza, Cardoso e Miranda (2016), há uma diversidade de fatores que podem alterar as possiblidades quanto ao aprender, assim ao analisar a aprendizagem no meio universitário, devem ser consideradas questões que extrapolam a responsabilização do indivíduo quanto aos seus resultados, devendo ser respeitados os recursos intelectuais dispostos pelo aluno, da mesma forma que a influência dos aspectos afetivos, sociais e físicos.

É crescente a discussão acerca da saúde mental do aluno universitário (Brandtner \& Bardagi, 2009), na mesma proporção é percebido no meio acadêmico o aumento das dificuldades de aprendizagem, assim como de leitura, deste modo foram analisados a correlação entre a intensidade dos sintomas de depressão por meio do BDI II e as habilidades em compreensão em leitura no teste de Cloze.

Neste trabalho, os resultados mostram que entre os alunos concluintes, foi encontrada correlação inversa estatisticamente significativa $(r=-0,3)$ entre o BDIl e o Cloze, indicando que os sintomas de depressão mental exercem influência sobre as habilidades em compreensão de leitura, pois quanto maior a intensidade dos sintomas de depressão apresentado pelo indivíduo, menor foi sua pontuação quanto as habilidades em compreensão de leitura.

Os resultados aqui apresentados evidenciam que a compreensão em leitura é influenciada pelas condições de saúde mental do aluno, que consequentemente irá influenciar em sua aprendizagem, visto a importância da leitura neste processo. Faz-se importante ressaltar que a compreensão em leitura transcende mecanismos cognitivos, envolvendo também os afetivos. Visto que a depressão é um transtorno de ordem afetiva, sua sintomatologia pode interferir na funcionalidade também no âmbito acadêmico, por meio de prejuízos nas habilidades em leitura, que consequentemente irão interferir em sua aprendizagem.

Tendo sido encontrada correlação entre os resultados do BDI II e o Cloze, é possível que a depressão também pode interferir em outras habilidades, como em que transtornos de ordem afetiva tendem a influenciar os processos cognitivos.

Todas estas questões apontam para a necessidade do desenvolvimento de programas na universidade voltados à Saúde Mental e também à aprendizagem do aluno universitário, porém não 
só com enfoque institucional, mas também clínico, respeitando o sofrimento psíquico do aluno, assim como suas dificuldades frente ao aprender.

A prevalência dos sintomas de ansiedade e depressão expressiva, mostram a carência de mais atenção e promoção à saúde mental dos estudantes (Fernandes, Vieira, Silva, Avelino, \& Santos, 2018). As instituições de ensino também devem exercer um papel social, sendo um ambiente propício à promoção e manutenção do bem-estar de seus membros, adotando ações sustentáveis e inovadoras, para que consigam ir ao encontro das especificidades que permeiam os determinantes de saúde da população universitária (Pina-Oliveira, 2017).

É importante que exista a sensibilização da universidade acerca da necessidade e relevância do aperfeiçoamento da prática docente, pois têm implicações diretas na formação de novos profissionais. Visto que a instituição, como um todo, deve atuar em prol do fortalecimento dos espaços e situações que estimulam o vínculo dos alunos com todo o cenário acadêmico, como espaços de acolhimento entre e para estudantes (Souza, Andrade \& Mancuso. 2020)

As universidades devem atuar como espaços de Promoção da Saúde, desenvolvendo ações voltadas às condições de vida do indivíduo por meio de um trabalho intersetorial, realizando parcerias com setores diversos das comunidades em que se encontram inseridas (Silva et al., 2019). Os ambientes e contextos que integram a vida do indivíduo e influenciam sua saúde e bemestar, são determinantes para a saúde, uma vez que, possibilitam ao indivíduo cuidar da própria saúde.

\section{CONSIDERAÇÕES FINAIS}

Foi evidenciado neste trabalho que os desequilíbrios de ordem subjetiva, derivados da depressão, tendem a prejudicar as habilidades em leitura do aluno universitário e, consequentemente, interferem em sua aprendizagem. Neste sentido, as condições de saúde mental do aluno, também são variáveis que interferem nas dificuldades de aprendizagem no ensino superior.

Diante dos resultados encontrados através desta pesquisa, é possível afirmar que a saúde mental fragilizada, com enfoque nos sintomas e transtornos depressivos, interfere de maneira negativa na aprendizagem dos alunos na etapa educacional universitária, de modo diretamente proporcional ao contexto do período escolar, conforme estudos publicados nos últimos anos.

Neste sentido, considera-se a preocupação em construção de espaços saudáveis que visem não apenas ações individualizadas direcionadas ao aluno com baixo rendimento acadêmico, pois isto seria culpabilizar exclusivamente o indivíduo. Em consonância com a proposta da Organização Mundial de Saúde (OMS), esta pesquisa entende que, além da atenção ao sujeito, fomentar a 
construção de universidades promotoras da saúde é um caminho imprescindível para promover qualidade de vida aos alunos.

Quanto às limitações do estudo, a primeira refere-se à autoaplicabilidade dos instrumentos, podendo influenciar assim os resultados apresentados, pois depende do comprometimento dos participantes. Outro ponto a ser considerado é o tamanho da amostra, que pode ser ampliada em outros estudos. E finalmente, aqui estudou-se um curso, são cabíveis estudos em outros cenários pois os resultados podem ser diferentes em outros grupos de alunos universitários e outras instituições de ensino, com naturezas técnico-administrativas diferentes.

\section{REFERÊNCIAS}

Abreu, K. N. M., Garcia, D. C., Hora, K. F. P. N. A, \& Souza, C. R. (2017). O teste de Cloze como instrumento de medida da proficiência em leitura: fatores linguísticos e não linguísticos. Revista de Estudos da Linguagem, 25(3), 1767-1799. Doi: http://dx.doi.org/10.17851/22372083.25.3.1767-1799.

Almeida, R. P., Piza, C. J. M. T., Cardoso, T. S. G., \& Miranda, M. C. (2016). Prevenção e remediação das dificuldades de aprendizagem: adaptação do modelo de resposta à intervenção em uma amostra brasileira. Revista Brasileira de Educação, 21(66), 611-630. Doi: http://dx.doi.org/10.1590/S1413-24782016216632.

American Psychiatric Association. (2014). Manual diagnóstico e estatístico de transtornos mentais: DSM-5. Porto Alegre: Artmed.

Base Nacional Comum Curricular. (2017). Portal do Ministério da Educação (MEC) Web Site. Recuperado em 06 de maio, 2019, disponível em: http://basenacionalcomum.mec.gov.br.

Bolsoni-Silva, A. T., \& Guerra, B. T. (2014). O impacto da depressão para as interações sociais de universitários. Estudos e Pesquisas em Psicologia, 14(2), 429-452. Disponível em: http://pepsic.bvsalud.org/scielo.php?script=sci arttext\&pid=S180842812014000200004.

Borine, R. C. C, Wanderley, K. S., \& Bassit, D. P. (2015). Relação entre a qualidade de vida e o estresse em acadêmicos da área da saúde. Estudos Interdisciplinares em Psicologia, 6(1), 100118. Doi: 10.5433/2236-6407.2015v6n1p100.

Costa, G. P. (2015). A Clínica Psicanalítica das Psicopatologias Contemporâneas-2. Porto Alegre: Artmed.

Brandtner, M., \& Bardagi, M. (2009). Sintomatologia de depressão e ansiedade em estudantes de uma universidade privada do Rio Grande do Sul. Gerais: Revista Interinstitucional de Psicologia, 2(2), 81-91. Disponível em http://pepsic.bvsalud.org/scielo.php?script=sci arttext\&pid=S1983$82202009000200004 \& \operatorname{lng}=\mathrm{pt} \& \operatorname{ting}=\mathrm{pt}$

Estrada Aranda, B. D., Álvarez, C. D., Hernández, R. L., \& Ramírez, M. T. Z. (2015). Propiedades psicométricas del modelo bifactorial del BDI II (versión española) em muestras mexicanas de poblacióna general y estudiantes universitários. Universitas Psychologica, 14(1), 125-136. Disponível em: https://revistas.javeriana.edu.co/index.php/revPsycho/article/view/5286. 
Fernandes, M. A., Vieira, F. E. R., Silva, J. S., Avelino, F. V. S. D., \& Santos, J. D. M. (2018). Prevalência de sintomas ansiosos e depressivos em universitários de uma instituição pública. Revista Brasileira de Enfermagem, 71(Supl. 5), 2169-2175. https://doi.org/10.1590/0034-71672017-0752

Finger, I. R., \& Argimon, I. I. L. (2013). Propriedades Psicométricas do Inventário de Depressão de Beck-II (BDI-II) em uma Amostra Universitária. Revista de Psicologia da IMED, 5(2), 383-392. Doi: http://dx.doi.org/10.18256/2175-5027/psico-imed.v5n2p84-91.

Fórum Nacional de Pró-Reitores de Assuntos Comunitários e Estudantis - FONAPRACE. (2014). IV Pesquisa do Perfil Socioeconômico e Cultural dos Estudantes de Graduação das Instituições Federais de Ensino Superior Brasileiras. Disponível em: http://www.andifes.org.br/wpcontent/uploads/2017/11/Pesquisa-de-Perfil-dos-Graduanso-das-IFES_2014.pdf.

Garcia, N. R., \& Boruchovitch, E. (2015). As atribuições de causalidade no ensino fundamental: relações com variáveis demográficas e escolares. Psico, 46(2), 176-187. Doi: http://dx.doi.org/10.15448/1980-8623.2015.2.17642.

Gorestein, C., \& Wang, Y. (2016). Inventário de Depressão de Beck (BDI). In: C. Gorestein, Y. Wang, \& I. Hungerbühler. (orgs.). Instrumentos de avaliação em saúde mental. Porto Alegre: Artmed.

Moreira, B. B. G., Martins-Reis, V. O., \& Santos, J. N. (2016). Autopercepção das dificuldades de aprendizagem de estudantes do ensino fundamental. Audiology, Communication, Research, 21, e1632. Doi: http://dx.doi.org/10.1590/2317-6431-2015-1632.

Papalia, D. E., Olds, S. W., \& Feldman, R. D. (2013). Desenvolvimento humano, (12a ed.). Porto Alegre: Artmed.

Paula, J. A., Borges, A. M. F. S., Bezerra, L. R. A., Parente, H. V., Paula, R. C. A., Wajnsztejn, R., Carvalho, A. A. S., Valenti, V. E., \& Abreu, L. C. (2014). Prevalência e fatores associados à depressão em estudantes de medicina. Journal of Human Growth and Development, 24 (3), 274-281. Disponível em: http://www.revistas.usp.br/ihgd/article/view/88911.

Pina-Oliveira, A. A. (2017). Promoção da Saúde e o imperativo do conhecimento para a ação. Revista Saúde, 11(1-2), 04-05. Disponível em: https://www.researchgate.net/publication/321945749 Promocao da Saude e o imperativo do conhecimento para a acao.

Silva, M. R. I., Almeida, A. P. de, Machado, J. C., Silva, L. S. da, Cardoso, J. A. F., Costa, G. D., \& Cotta, R. M. M. (2019). Processo de Acreditação das Escolas Promotoras de Saúde em âmbito mundial: revisão sistemática. Ciência e Saúde Coletiva, 24(2), 475-486. Doi: https://dx.doi.org/10.1590/1413-81232018242.23862016.

Souza, G. G. O., de Andrade, E. A., \& Mancuso, A. M. C. (2020). Vivência universitária e rendimento acadêmico de graduandos em nutrição egressos de escolas públicas. Cadernos da FUCAMP, 19(37), 1-20.

Sternberg, R. J. (2012). Psicologia Cognitiva. São Paulo: Cengage Learning.

Tanzawa, E. C. L., \& Pullin, E. M. M. P. (2012). Leituras prescritas e práticas de leitura de estudo no ensino superior. Psicologia Escolar e Educacional, 16(2), 265-274. Doi: http://dx.doi.org/10.1590/S1413-85572012000200009. 
World Health Organization. (2017). Depression and Other Common Mental Disorders: Global Health Estimates Web site. Recuperado em 06 maio de 2019. Disponível em: http://apps.who.int/iris/bitstream/handle/10665/254610/WHO-MSD-MER-2017.2-eng.pdf. 